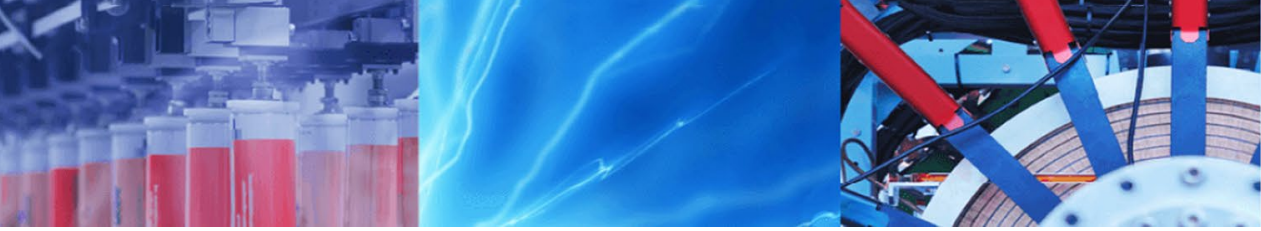

Research Article

\title{
Gas pressure and density effects on vibration of cylindrical pressure vessels: analytical, numerical and experimental analysis
}

\author{
Omid Mir $^{1} \cdot$ Meisam Shakouri ${ }^{1} \cdot$ M. R. Ashory ${ }^{2}$
}

Received: 10 October 2019 / Accepted: 17 December 2019 / Published online: 23 December 2019

(c) Springer Nature Switzerland AG 2019

\begin{abstract}
The present research deals with the natural frequencies and mode shapes of cylindrical pressure vessels filled with different gasses in various internal pressures. The Hamilton's principle is employed to derive the governing equations, which are solved using analytical and finite element analyses, and a closed form relation as the equivalent density is obtained to include the gas properties. The experimental modal analysis is performed and the obtained results are compared and validated with analytical and finite element results. The results show that the type of the gas, as well as the gas pressure have significant effect on vibrational behavior of the structure and should be accounted in the design and analysis of vessels.
\end{abstract}

Keywords Free vibration · Modal testing · Pressure vessel

\section{Introduction}

Pressure vessels have many applications in different areas of engineering like mechanical, aerospace, marine, etc. Since the pressure vessels should work with various gas types and pressures during the working life, the effects of gas properties on the operation of the vessel should be considered in the design process.

Many researchers previously studied the vibration characteristics of cylindrical pressure vessels with internal and external pressure [1-6]. Li et al. [7] analyzed the effects of hydrostatic pressure on the vibration of piezoelectric laminated cylindrical shell. Senjanović et al. [8-10] studied the effects of internal pressure on vibrational behavior of rotating cylindrical shell. Arnold and Warburton [11, 12] employed Hamilton's principle to derive equations of motion of the cylindrical shell. Amabili et al. [13] investigated the vibration of simply supported cylindrical shell containing an incompressible fluid using Donnell shallow shell theory. Fluid-filled cylindrical shell subjected to lateral harmonic loading is studied by Del Prado et al. [14]. In addition, Zhang et al. [15] considered vibration of composite cylindrical shells subjected to axial pressure and radial load in both side of the cylinder. Bolotin [16] focused on the natural frequency distribution and the intervals in cylindrical shells and Fung et al. [17] examined the effects of shell thickness and internal pressure on the vibration of cylinders. Analytical and experimental study is done by Stillman [18] to describe the vibration behavior of liquidfilled pressure vessels. Selmane and Lakis [19] presented the vibration of anisotropic cylindrical shell subjected to internal and external flow using Sanders shell theory. The generalized Fourier series are used by Stepanishen [20] to evaluate fluid pressure effect on vibratory response of cylindrical shells with infinite rigid extensions. Chiba and Abe [21] analyzed hydroelastic vibration of cylindrical vessel containing liquid and Gupta and Hutchinson [22] studied the free vibration of liquid storage tanks. Krishna and Ganesan [23] introduced an approach based on polynomial terms for calculating the added mass for fluid-filled

Meisam Shakouri, shakouri@semnan.ac.ir| 'Department of Aerospace Engineering, Semnan University, P.O. Box 35131-19111, Semnan, Iran. ${ }^{2}$ Department of Mechanical Engineering, Semnan University, P.O. Box 35131-19111, Semnan, Iran. 
cylindrical shells. The vibration of partially filled laminated composite cylindrical shells is studied in ref [24]. Isvandzibaei et al. [25] studied the effects of internal pressure in cylindrical shells made of functionally graded materials (FGMs) using first order shear deformation theory. Shakouri et al. [26] studied the Effects of imperfection shapes on buckling of conical shells under compression.

According to the above papers, many of previous investigations of cylindrical shell vibrations have been limited to unpressurized vessels. In addition, in the case of pressurized vessels, the pressure is applied as the internal loading on the shell and the effects of the gas properties on the vibration of the pressure vessel are not considered.

In this paper, the natural frequencies and mode shapes of pressurized cylinders containing various types of the gasses (air and oxygen) with internal pressures ranges from zero to 100 bar is investigated. The total kinematic energy of the cylinder and internal gasses has been written and the governing equation is obtained using Hamilton's equation and the relation for natural frequencies of the pressure vessel including gas effects is obtained. The results are compared and validated with finite element analysis and modal testing. Finally, the effects of mass and pressure of the gas on the frequency response function of the pressurized cylinder is studied.

\section{Governing equations for pressure vessel containing gas}

\subsection{Displacements and strains}

Figure 1 illustrates the cylindrical pressure vessel considered in this paper. The $(r, \theta, z)$ represent the cylinder in radial, circumferential and longitudinal directions, respectively. It is assumed that the cylinder remains circular in all internal pressures. Thus, the displacements of the shell can be shown as [27]:

$\delta r=A \cos (\beta \theta) \sin \left(\frac{\alpha \pi z}{L}\right)$

$\delta \theta=\delta z=0$

where $\delta r, \delta \theta, \delta z$ denotes radial, circumferential and axial displacement, $L$ represents length, $r$ is the radius, $t$ is the
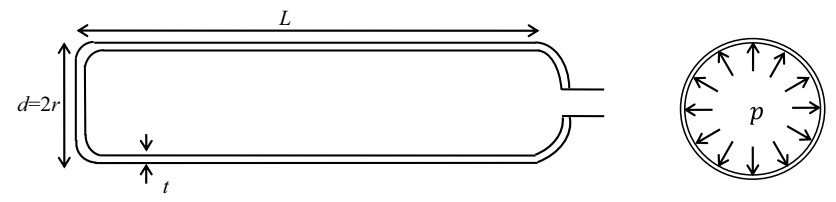

thickness and $A$ is the amplitude of radial displacement which is periodic over time.

$A=A_{0} \sin \omega t$

In addition, $\alpha, \beta$ are axial half wave and circumferential wave, respectively.

\subsection{Governing equations}

To extract the governing equation, bending and stretching energies are written for pressurized cylindrical shell. Fluid expansion energy is represented for indicating fluid effect. Tensile tension in cylindrical shell due to internal gas pressure is $\frac{p r}{2}$ and $p r$ in the axial and circumferential directions, respectively. For deformation of the middle surface in $\theta$ direction we have [27]

$\Delta \theta=\int_{0}^{2 \pi}\left(\sqrt{r^{2}+2 \delta r . r+\delta r^{2}+\left(\frac{\partial \delta r}{\partial \theta}\right)^{2}}-r\right) d \theta$

where $\Delta \theta$ denotes the circumferential expansion of the cylinder. Considering small displacements (i.e. $\delta r / r \ll 1, \partial \delta r / r \partial \theta \ll 1)$, the Eq. (3) can be written as

$\Delta \theta=\int_{0}^{2 \pi}\left(\sqrt{\delta r+\frac{1}{2 r}\left(\frac{\partial \delta r}{\partial \theta}\right)^{2}}-r\right) d \theta$

$\Delta z=\frac{1}{2} \int_{0}^{2 \pi}\left(\frac{\partial \delta r}{\partial z}\right)^{2} d z$

where $\Delta z$ is the axial expansion of the cylindrical shell. The stretching energy $\left(V_{S}\right)$ of the cylinder can be written as [27]

$V_{s}=\int_{0}^{2 \pi} \int_{0}^{x} p\left[\delta r+\frac{1}{2 r}\left(\frac{\partial \delta r}{\partial \theta}\right)^{2}\right] r d \theta d z+\frac{1}{4} \int_{0}^{2 \pi} \int_{0}^{x} p r^{2}\left(\frac{\partial \delta r}{\partial z}\right)^{2} d \theta d z$

where $p$ is internal pressure of the cylinder. In addition, the gas expansion energy $\left(V_{E}\right)$ is

$V_{E}=-\int_{0}^{2 \pi} \int_{0}^{x} p r \delta r d \theta d z$

Combining Eqs. (6) and (7) results in

$V_{S}+V_{E}=\frac{1}{2} \int_{0}^{2 \pi} \int_{0}^{x} \frac{p}{r}\left[\left(\frac{\partial \delta r}{\partial \theta}\right)^{2}+\frac{r^{2}}{2}\left(\frac{\partial \delta r}{\partial z}\right)^{2}\right] r d \theta d z$

Fig. 1 Schematic of cylindrical pressure vessel 
$V_{S}+V_{E}=\frac{1}{4}\left[\pi p x(A \beta)^{2}\left(1+\frac{\lambda^{2}}{\beta^{2}}\right)\right]$

$\lambda=\frac{\pi r \alpha}{x}$

The bending energy $\left(V_{B}\right)$ of cylinder can be defined as

$V_{B}=\int_{0}^{2 \pi} \int_{0}^{x} \int_{-t / 2}^{t / 2} \frac{E r}{2\left(1-v^{2}\right)}\left[\varepsilon_{z}^{2}+\varepsilon_{\theta}^{2}+2 v \varepsilon_{z} \varepsilon_{\theta}+\left(1-\frac{v}{2}\right) \tau_{\theta z}^{2}\right] d \theta d z d x$

where $E$ is young modulus and $v$ is the Poisson's ratio. In addition, $\varepsilon_{r}, \varepsilon_{\theta}, \varepsilon_{z}$ represent the strains in radial, circumferential and axial directions, respectively and $\tau_{\theta z}$ is the shear strain. According to [28], by thin shell assumption for the cylinder, the values of normal stress in radial direction and the shear strain can be ignored. Therefore, we have

$\varepsilon_{z}=-\mu\left(\frac{\partial^{2} \delta r}{\partial^{2} z}\right)=\left(\frac{\alpha \pi}{x}\right)^{2} A \mu \cos (\beta \theta) \sin \left(\frac{\alpha \pi z}{x}\right)$

$\varepsilon_{\theta}=-\mu\left(\frac{\partial^{2} \delta r}{\partial^{2} \theta}\right)=\left(\frac{\beta}{r}\right)^{2} A \mu \cos (\beta \theta) \sin \left(\frac{\alpha \pi z}{x}\right)$

Substituting Eqs. (12) and (13) into (11), the potential bending energy of cylinder can be expressed as

$V_{B}=\frac{\pi x E A^{2} t^{3}\left(\lambda^{2}+\beta^{2}\right)^{2}}{48 r^{3}\left(1-v^{2}\right)}$

The kinetic energy of the cylinder is considered as

$T_{C}=\frac{1}{2} \int_{0}^{2 \pi} \int_{0}^{x^{r+}} \int_{t_{1 / 2}}^{r+2} \rho_{C} r\left(\frac{\partial \delta r}{\partial T}\right)^{2} d \theta d z d r=\frac{1}{4} \pi r x t \rho_{C} \dot{A}^{2}$

where $T_{C}$ and $\rho_{C}$ respectively denote the kinetic energy and density of the cylinder. The gas in the cylinder is assumed incompressible and non-rotating, only with small pressure variations. According to these assumptions, for kinetic energy of the pressurized gas, we have

$T_{G}=\frac{1}{2} \rho_{G} \int_{0}^{x} \int_{0}^{2 \pi} r V_{G} \frac{\partial V_{G}}{\partial H} d \theta d z$

where $T_{G}$ represents the kinetic energy of gas, $\rho_{G}$ represents the density of gas, $V_{G}$ is the velocity potential and $H$ is the unit vector, normal to the cylinder surface. For small displacements we have $\frac{\partial V_{G}}{\partial H} \approx \frac{\partial V_{G}}{\partial r}$

$t \ll r$

$T_{G}=\frac{1}{2} \rho_{G} \int_{0}^{x} \int_{0}^{2 \pi} r V_{G} \frac{\partial V_{G}}{\partial r} d \theta d z$

To satisfy the Hamilton's principle, the velocity potential can be assumed as

$V_{G}=-\frac{x \dot{A} I_{n}\left(\frac{\alpha \pi r}{x}\right)}{\pi \alpha l_{n}^{\prime}\left(\frac{\alpha \pi r}{x}\right)} \sin \left(\frac{\alpha \pi z}{x}\right) \cos (\beta \theta)$

$-\frac{\partial V_{G}}{\partial r}(r, \theta, z)=\dot{A} \sin \left(\frac{\alpha \pi z}{x}\right) \cos (\beta \theta)$

where $I_{n}$ is the modified Bessel function. By substituting Eq. (20) into (19), the kinetic energy of the gas can be expressed as

$T_{G}=\frac{\pi \rho_{G} \dot{A}^{2} x^{3} I_{n}\left(\frac{\alpha \pi r}{x}\right)}{4 \alpha \pi I_{n}^{\prime}\left(\frac{\alpha \pi r}{x}\right)}$

where the expressions of Modified Bessel function can be explained as [29]

$I_{n}^{\prime}(y)=I_{n-1}(y)-\frac{n}{z} I_{n}(y)$

$I_{n}(y)=\frac{\left(\frac{y}{2}\right)^{n}}{\Gamma(n+1)}$

where $n$ and $y$ are general parameters and $\Gamma$ denote the gamma function. According to Eqs. (23)-(24) and (22), the potential energy of gas can be obtained as

$T_{G}=\frac{\pi r x^{2} \rho_{G} \dot{A}^{2}}{4 n}$

Therefore, the total kinetic energy of system is obtained as

$T_{T}=T_{C}+T_{G}=\frac{1}{4} \pi r x t \rho_{E} \dot{A}^{2}$

Where $T_{T}$ denotes total kinetic energy, $\rho_{E}$ is an equivalent density as follows

$\rho_{E} \equiv \rho_{C}+\frac{r \rho_{G}}{n t}$ 
Using Eq. (14) and (9) the total potential energy of the system can be written as

$V_{T}=\frac{\pi \times A\left[E t^{3}\left(\lambda^{2}+\beta^{2}\right)^{2}+12 p r^{3}\left(\beta^{2}+\lambda^{2}-v^{2}\left(\beta^{2}+\lambda^{2}\right)\right)\right]}{48\left(1-v^{2}\right) r^{3}}$

which $V_{T}$ represents the total potential energy. Considering the Lagrange equation for investigating natural frequencies [30]

$\frac{d}{d t}\left(\frac{\partial T_{T}}{\partial \dot{q}}\right)-\frac{\partial T_{T}}{\partial q}=-\frac{\partial V_{T}}{\partial q}$

The natural frequency of cylinder can be expressed as

$\omega=\omega_{0} \sqrt{\frac{\rho_{C}}{\rho_{E}}+\frac{12 p r^{3}\left(1-v^{2}\right)}{E t^{3}\left(\lambda^{2}+\beta^{2}\right)}}$

where $\omega$ represents natural frequency of pressurized cylindrical shell and $\omega_{0}$ is the natural frequency of the cylinder, without considering the gas effects.

Table 1 Measured geometry and material properties of pressure vessel

\begin{tabular}{llll}
\hline Material & \multicolumn{3}{l}{ Geometry } \\
\hline Density & $7850\left(\mathrm{~kg} / \mathrm{m}^{3}\right)$ & $L$ & $41.5 \mathrm{~cm}$ \\
Elastic modulus & $207,000(\mathrm{MPa})$ & $r$ & $7 \mathrm{~cm}$ \\
Poisson's ratio & 0.286 & $t$ & $4.7 \mathrm{~mm}$ \\
Shear modulus & $80,482(\mathrm{MPa})$ & & \\
Mass of cylinder & $9.48(\mathrm{~kg})$ & & \\
\hline
\end{tabular}

\section{Experimental study}

To validate the accuracy of Eq. (30), the pressurized cylinder made of steel is tested in modal laboratory. Material and geometrical properties of the pressure vessel are presented in Table 1. Figure 2 shows the test equipment and pressure vessel in modal lab.

Oxygen and air gasses are filled separately into the cylinder. These gasses have different molar mass, which cause their densities to be different. Table 2 shows molar mass of oxygen and air.

\subsection{Supports}

The cylinder is free in both sides and the first six frequencies belongs to the rigid body motions (i.e. the frequency is zero). To obtain the free boundary condition, the pressure vessel is hanged with a long rope, so that the frequencies implemented from the boundary conditions are far from the vessel frequencies [31].

\subsection{Excitation and accelerometers}

The 4-input channel analyzer is used and the motion is detected and measured by using piezoelectric accelerometer. Number of points which be created in cylinder for modal testing is 49 points and the roving hammer method is used to excite the cylinder. Each frequency response

Table 2 Molar mass of oxygen and air

\begin{tabular}{ll}
\hline Gas type & Molar mass \\
\hline Oxygen & $31.998\left(\frac{\mathrm{gr}}{\mathrm{mol}}\right)$ \\
Air & $28.966\left(\frac{\mathrm{gr}}{\mathrm{mol}}\right)$ \\
\hline
\end{tabular}

Fig. 2 Test equipment's and cylinder in modal lab
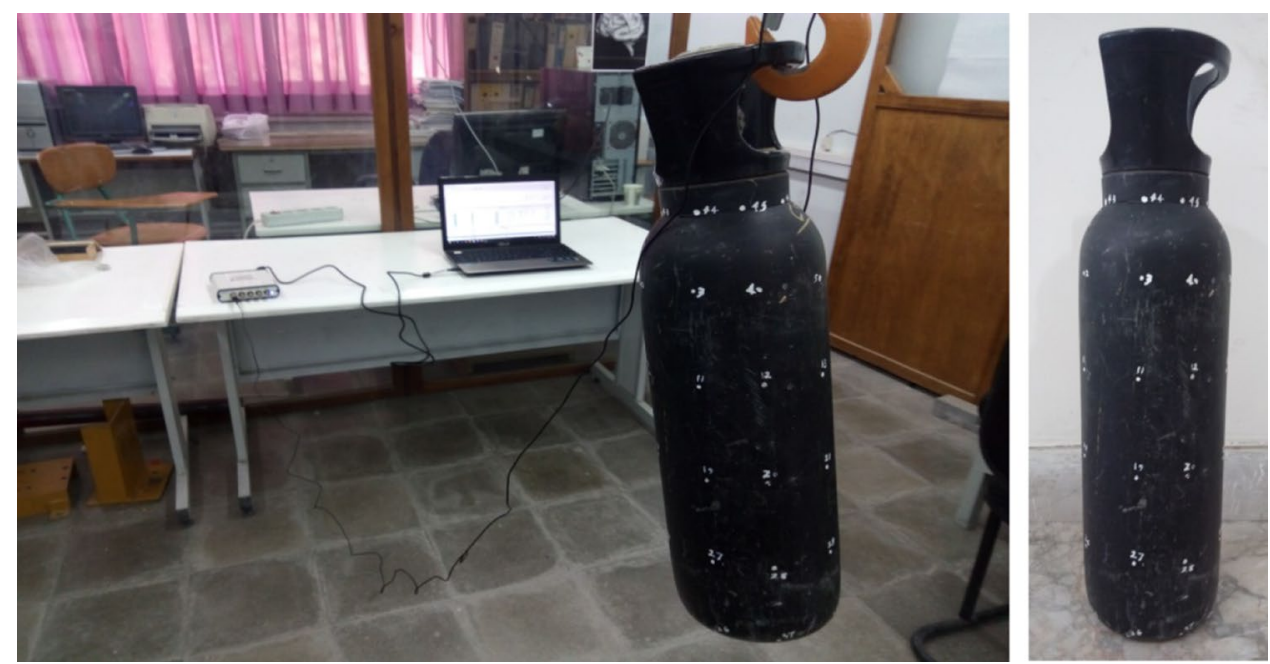
function (FRF) is evaluated after averaging over several measurements (three times) in order to reduce noise.

\section{Finite element analysis}

For finite element (FE) analysis, the traditional ANSYS software is used and the 4-node SHELL181 element is employed to model the vessel. The Lanczos method is employed to obtain the natural frequencies and mode shapes. The pressure is applied as internal distributed load on the shell and the effects of the gas mass is modeled by using the equivalent density as presented in Eq. (27). After the mesh study and convergence check, the total number of mesh elements in cylindrical shell is 55376, which is the optimum conditions between solution time and the accuracy of the results.

\section{Results and discussion}

In this section, numerical, analytical and experimental results are presented and compared for the vibration of pressurized vessel. The pressure changes from zero to 100 bars and two types of gasses including oxygen and air are used. Effect of gas pressure on cylinder shell in the simulation is assumed steady. Tables 3 and 4 represent the first frequencies (excluding rigid body frequencies) of the system for air and oxygen, respectively. These frequencies are related to pressure and gas, which are calculated, by experiment, FE and analytical methods. In addition, the frequencies of pressurized vessels without considering the gas density effects is investigated. It can be seen that the results of Eq. (30) are in good accordance with finite element and experiment. In addition, the gas density has significant effect on the natural
Table 3 Comparison of natural frequencies of cylinder which filled by air in various pressures

\begin{tabular}{lllll}
\hline \multicolumn{2}{l}{ Cylinder filled with air } & & & \\
\hline Pressure (Bar) & Experiment $(\mathrm{Hz})$ & $\begin{array}{l}\text { FE with mass } \\
\text { effect }(\mathrm{Hz})\end{array}$ & $\begin{array}{l}\text { FE without mass } \\
\text { effect }(\mathrm{Hz})\end{array}$ & Equation (30) $(\mathrm{Hz})$ \\
\hline 0 & 1136.9 & 1135.5 & 1135.5 & 1135.9 \\
10 & 1146.4 & 1143.0 & 1153.3 & 1140.4 \\
20 & 1150.0 & 1146.8 & 1162.7 & 1144.9 \\
30 & 1154.1 & 1150.5 & 1171.4 & 1149.4 \\
40 & 1158.5 & 1153.2 & 1180.5 & 1154.0 \\
50 & 1161.8 & 1156.1 & 1186.2 & 1158.5 \\
60 & 1169.6 & 1162.6 & 1200.0 & 1163.0 \\
70 & 1171.6 & 1163.8 & 1205.6 & 1167.6 \\
80 & 1177.5 & 1164.6 & 1216.4 & 1172.1 \\
90 & 1180.8 & 1165.5 & 1223.3 & 1176.6 \\
100 & 1186.6 & 1169.8 & 1232.9 & 1181.2 \\
\hline
\end{tabular}

Table 4 Comparison of natural frequencies of cylinder which filled by oxygen in various pressures

\begin{tabular}{lllll}
\hline \multicolumn{2}{l}{ Cylinder filled with oxygen $(\mathrm{Hz})$} & & \\
\hline Pressure (Bar) & Experiment $(\mathrm{Hz})$ & $\begin{array}{l}\text { FE with mass } \\
\text { effect }(\mathrm{Hz})\end{array}$ & $\begin{array}{l}\text { FE without mass } \\
\text { effect }(\mathrm{Hz})\end{array}$ & Equation $(30)(\mathrm{Hz})$ \\
\hline 0 & 1136.9 & 1135.5 & 1135.5 & 1135.9 \\
10 & 1144.3 & 1138.6 & 1153.3 & 1139.0 \\
20 & 1145.8 & 1140.1 & 1162.7 & 1142.2 \\
30 & 1149.6 & 1141.8 & 1171.4 & 1145.3 \\
40 & 1152.7 & 1143.5 & 1180.5 & 1148.5 \\
50 & 1155.7 & 1146.5 & 1186.2 & 1151.6 \\
60 & 1160.3 & 1152.3 & 1200.0 & 1154.8 \\
70 & 1163.1 & 1153.9 & 1205.6 & 1157.9 \\
80 & 1166.8 & 1156.3 & 1216.4 & 1161.4 \\
90 & 1170.3 & 1158.6 & 1223.3 & 1164.2 \\
100 & 1173.5 & 1160.6 & 1232.9 & 1167.4 \\
\hline
\end{tabular}


frequency, where the obtained results without gas density are far from the experiment results.

As the Tables 3 and 4 show, the density of the gas can change the value of frequencies, so in order to obtain exact frequency values of the pressurized vessel, the type of the gas must be denoted. Figure 3 shows the frequency response function as well as the phase angle of the pressurized cylinder with different pressures for both air and oxygen. As can be seen, except the third natural frequency, the natural frequencies of system increase with increase in the pressure. The remarkable phenomena, which is observed in both air and oxygen gasses, is that the third natural frequency decreases with increase in the internal pressure.
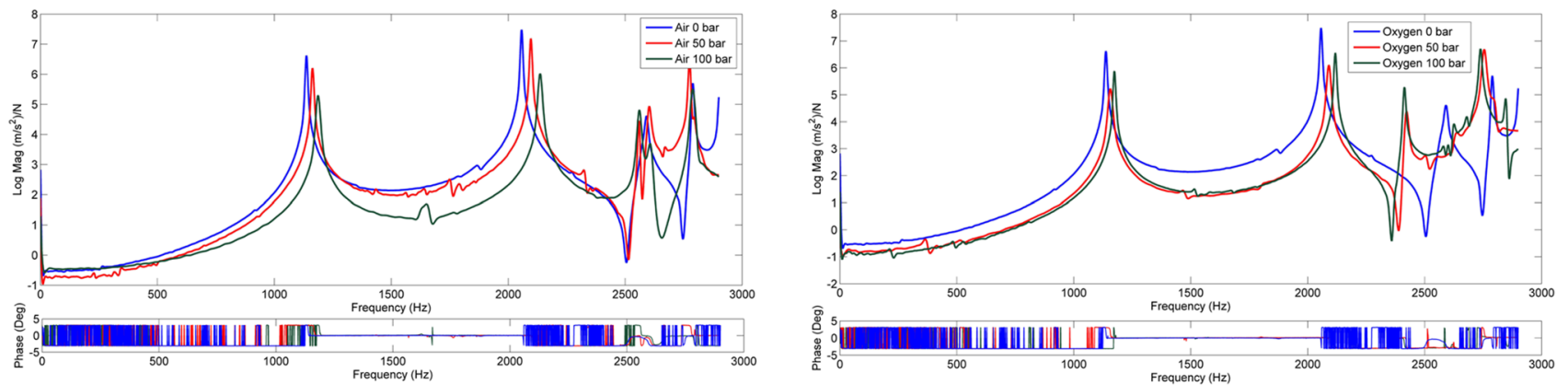

Fig. 3 Frequency response function of cylinder for 0,50 , and 100 bar inlet pressure

Fig. 4 Compare Frequency response function of cylinder filled with air and oxygen in 100 bar inlet pressure
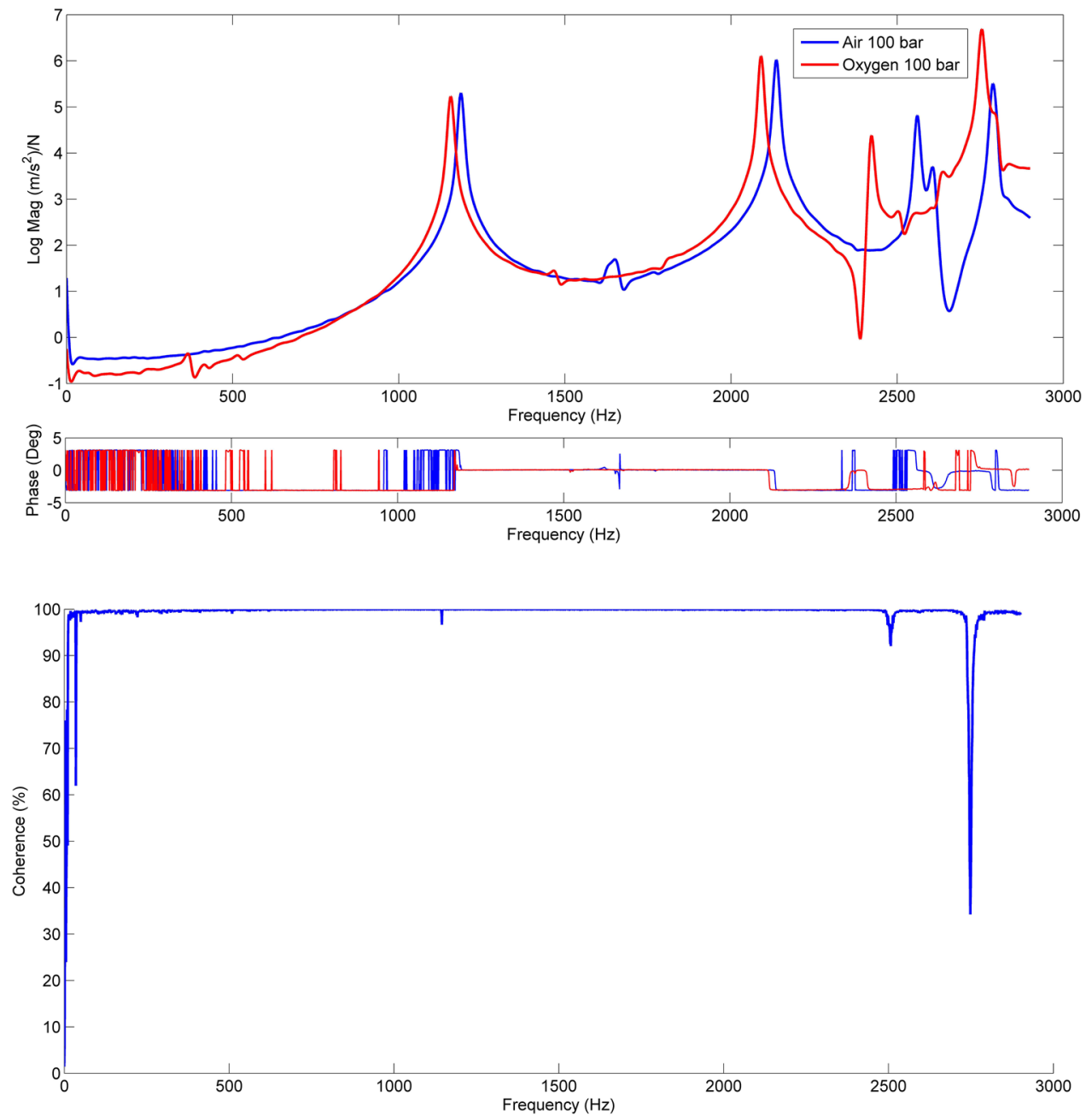
Fig. 5 Compare frequencies regression in various bar inlet pressure

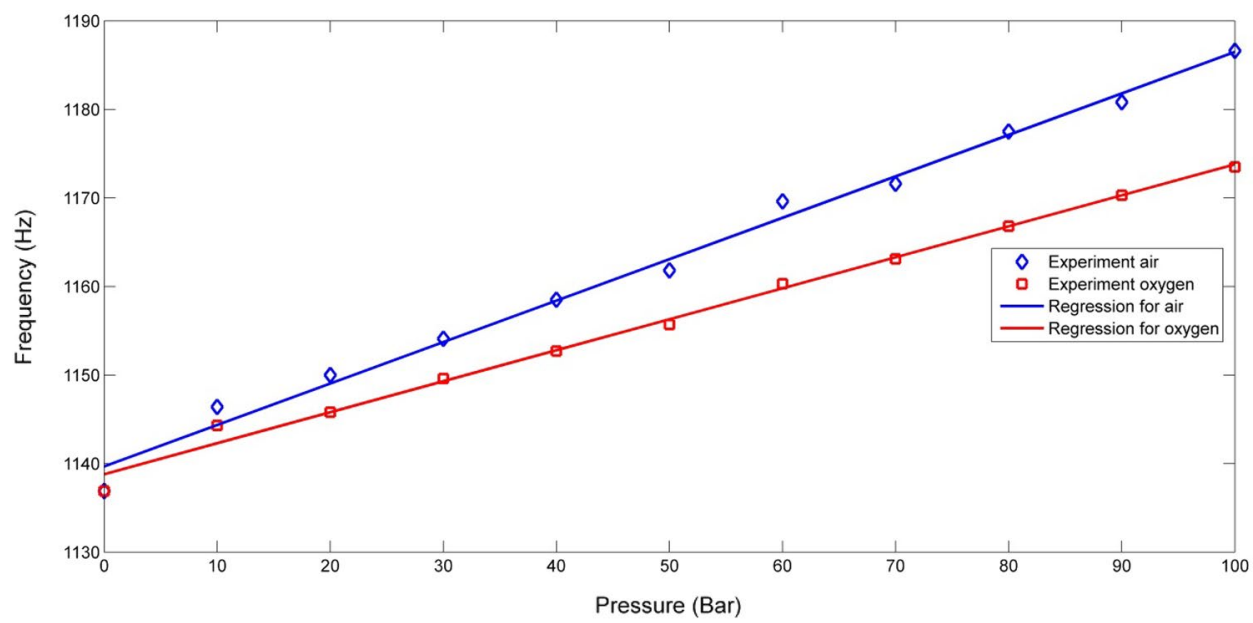

Figure 4 shows the frequency response function and corresponding phase angles of the pressurized cylinder filled with oxygen and air in the same pressure. This figure apparently describes the effects of gas density on the natural frequencies of the pressure vessel. As can be seen, since the stiffness of the vessel is the same for both gasses, it is expected that the air with less mass density has the higher natural frequency.

Results of Fig. 4 explain that density of gas can change natural frequencies and if type of gas is ignored in FE simulation, the results are different from the experiment. Figure 5 represents the variation of the frequency with respect to pressure, obtained from the experiment and corresponding linear fit (regression) between frequency and pressure. The linear function for both gasses are

$\omega=0.468 p+1139.677 \quad$ (Air)

$\omega=0.35 p+1138.791 \quad$ (Oxygen)

It can be seen that each gas causes different slope in frequency-pressure relation.

The first three mode shapes extracted from FE analysis and modal test are compared in Fig. 6 . As can be seen the obtained mode shapes are in good accordance with the numerical results.

To ensure from the independency of the mode shapes from each other, the auto-MAC study of the mode shapes are plotted Fig. 7. As seen, the mode shapes are independent from each other and none of them has significant effect on the others.

\section{Conclusions}

In this paper, the natural frequencies and mode shapes of pressurized cylinder considering the effects of pressure and the kind of the gas are studied. The modal analysis is done for two gasses (air and oxygen) in order to measure the effects of gas density on dynamic parameters of pressure vessel. The governing equations are obtained by evaluating kinetic and potential energies of system and substituting them into Lagrange equation. The results are compared to modal results and finally the effect of pressure and density of gas in are investigated. The major findings are:

- The gas density has significant effect on the natural frequency of the system. The natural frequency of cylindrical shell increases with increase in the pressure.

- For the gas with higher density, the frequencies gradient decreases with respect to pressure. It is mainly because of the fact that the mass of the system has reverse effect on the natural frequency.

- Considering both pressure and gas effect on the frequency, the effect of pressure is more than the mass, so the frequencies of system increases by increasing pressure and mass.

- The mode shapes of the pressure vessel do not change with the pressure and density of the gas. 
mode

$1^{\text {st }}$

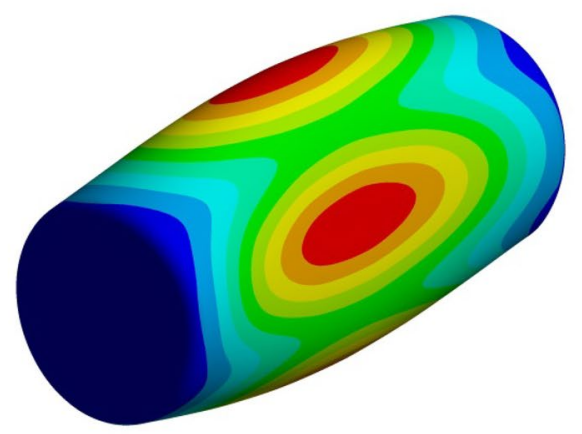

$2^{\text {nd }}$

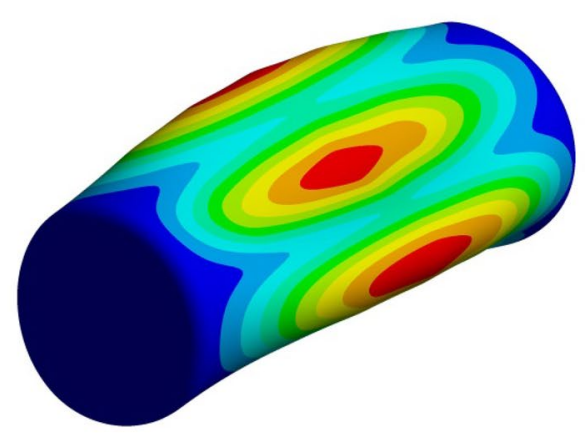

$3^{\text {rd }}$

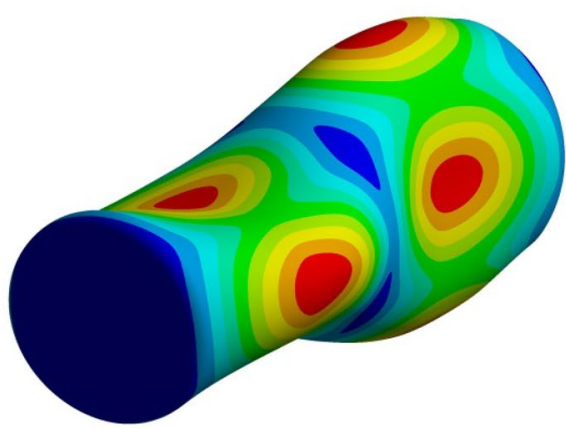

\section{Experiment}
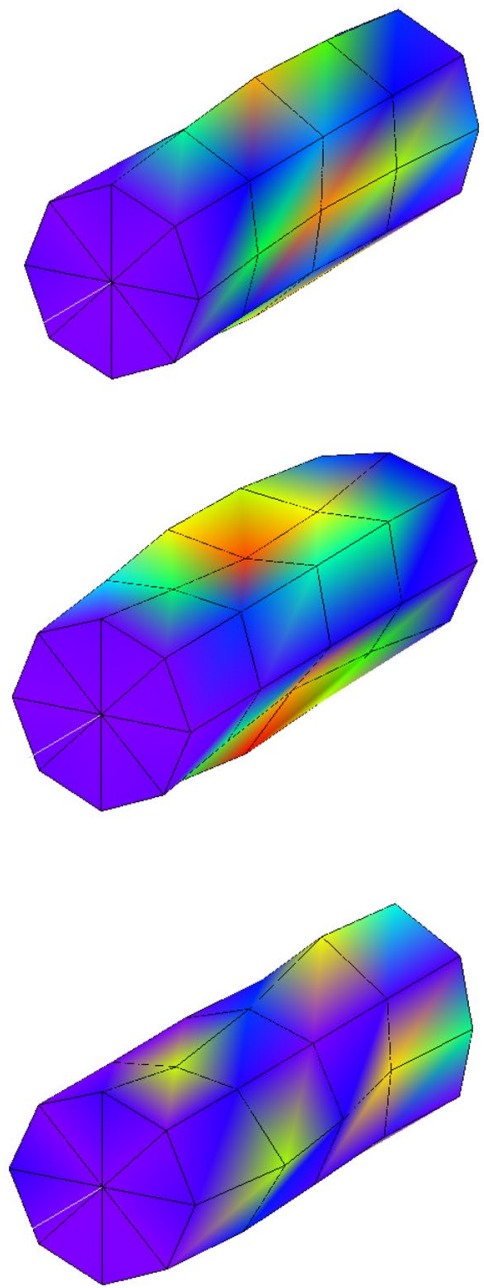

Fig. 6 Three mode shapes of cylinder extracted from FEM and modal testing

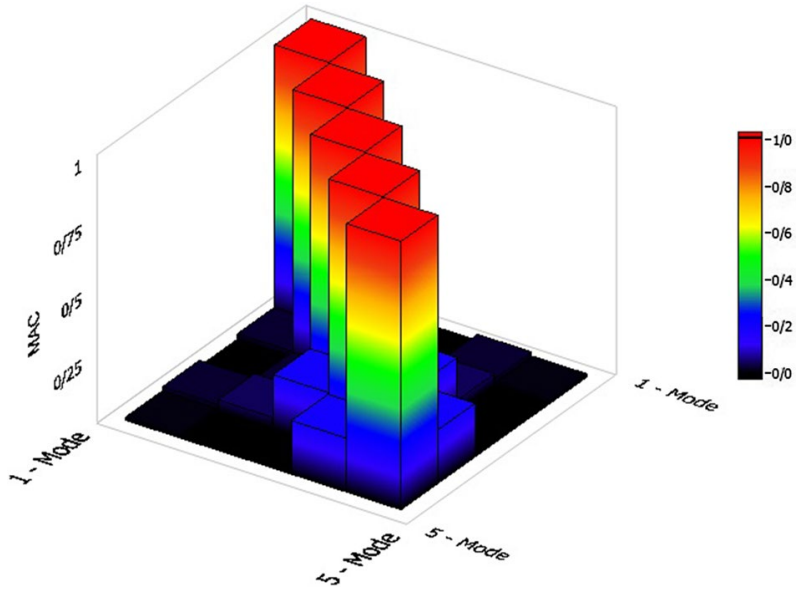

Fig. 7 Auto MAC for five mode shapes of cylinder extracted from modal testing

\section{SN Applied Sciences}

\section{Compliance with ethical standards}

Conflict of interest The authors declare that they have no conflict of interest.

\section{References}

1. Ross C, Haynes P, Richards WD (1996) Vibration of ring-stiffened circular cylinders under external water pressure. Comput Struct 60(6):1013-1019

2. Ross CTF, Haynes P, Richards WD (1996) Vibration of ring-stiffened circular cylinders under external water pressure. Comput Struct 60:1013-1019

3. Païdoussis MP, Suss S, Pustejovsky M (1977) Free vibration of clusters of cylinders in liquid-filled channels. J Sound Vib $55: 443-459$ 
4. Fuller CR, Fahy FJ (1982) Characteristics of wave propagation and energy distributions in cylindrical elastic shells filled with fluid. J Sound Vib 81:501-518

5. Kabir MZ (2000) Finite element analysis of composite pressure vessels with a load sharing metallic liner. Compos Struct 49:247-255

6. Pavlou DG (2016) Dynamic response of a multi-layered FRP cylindrical shell under unsteady loading conditions. Eng Struct 112:256-264

7. Li H, Lin Q, Liu Z, Wang C (2001) Free vibration of piezoelastic laminated cylindrical shells under hydrostatic pressure. Int $J$ Solids Struct 38:7571-7585

8. Senjanović I, Alujević N, Ćatipović I, Čakmak D, Vladimir N (2018) Vibration analysis of rotating toroidal shell by the Rayleigh-Ritz method and Fourier series. Eng Struct 173:870-891

9. Senjanović I, Ćatipović I, Alujević N, Vladimir N, Čakmak D (2018) A finite strip for the vibration analysis of rotating cylindrical shells. Thin-Walled Struct 122:158-172

10. Senjanović I, Áatipović I, Alujević N, Čakmak D, Vladimir N (2019) A finite strip for the vibration analysis of rotating toroidal shell under internal pressure. J Vib Acoust 141:021013

11. Arnold RN, Warburton GB (1949) Flexural vibrations of the walls of thin cylindrical shells having freely supported ends. Proc $R$ Soc Lond Ser A Math Phys Sci 197:238-256

12. Arnold RN, Warburton GB (1953) The flexural vibrations of thin cylinders. Proc Inst Mech Eng 167:62-80

13. Amabili M, Pellicano F, PaïDoussis MP (1998) Nonlinear vibrations of simply supported, circular cylindrical shells, coupled to quiescent fluid. J Fluids Struct 12:883-918

14. del Prado ZJ, Amabili M, Gonçalves PB (2017) Non linear vibrations of imperfect fluid-filled viscoelastic cylindrical shells. Procedia Eng 199:570-576

15. Zhang W, Yang S, Mao J (2018) Nonlinear radial breathing vibrations of CFRP laminated cylindrical shell with non-normal boundary conditions subjected to axial pressure and radial line load at two ends. Compos Struct 190:52-78

16. Bolotin VV (1963) On the density of the distribution of natural frequencies of thin elastic shells. J Appl Math Mech 27:538-543

17. Fung YC, Sechler EE, Kaplan A (1957) On the vibration of thin cylindrical shells under internal pressure. J Aeronaut Sci 24:650-660
18. Stillman WE (1973) Free vibration of cylinders containing liquid. J Sound Vib 30:509-524

19. Selmane A, Lakis AA (1997) Vibration analysis of anisotropic open cylindrical shells subjected to a flowing fluid. J Fluids Struct 11:111-134

20. Stepanishen PR (1982) Modal coupling in the vibration of fluidloaded cylindrical shells. J Acoust Soc Am 71:813-823

21. Chiba M, Abe K (1999) Nonlinear hydroelastic vibration of a cylindrical tank with an elastic bottom containing liquidanalysis using harmonic balance method. Thin-Walled Struct 34:233-260

22. Gupta RK, Hutchinson GL (1988) Free vibration analysis of liquid storage tanks. J Sound Vib 122:491-506

23. Krishna BV, Ganesan N (2006) Polynomial approach for calculating added mass for fluid-filled cylindrical shells. J Sound Vib 291:1221-1228

24. Saviz MR (2016) Coupled vibration of partially fluid-filled laminated composite cylindrical shells. J Solid Mech 8:823-839

25. Isvandzibaei MR, Jamaluddin H, Raja Hamzah RI (2016) Vibration analysis of supported thick-walled cylindrical shell made of functionally graded material under pressure loading. J Vib Control 22:1023-1036

26. Shakouri M, Spagnoli A, Kouchakzadeh M (2016) Effects of imperfection shapes on buckling of conical shells under compression. Struct Eng Mech 60:365-386

27. Warren $\mathrm{CH}$, Barbin A (1988) Vibration of pressurized cylinders. In: Army Missile Command Redstone Arsenal Al Guidance And Control Directorate

28. Sadd MH (2009) Elasticity: theory, applications, and numerics. Academic Press, Cambridge

29. Baricz Á, Maširević DJ, Pogány TK (2017) Series of Bessel and Kummer-type functions. Springer, Berlin

30. Craig RR, Kurdila AJ (2006) Fundamentals of structural dynamics. Wiley, New York

31. Ewins DJ (1984) Modal testing: theory and practice. Research Studies Press, Letchworth

Publisher's Note Springer Nature remains neutral with regard to jurisdictional claims in published maps and institutional affiliations. 